\title{
BMJ Open Phase 3 randomised trial of eltrombopag versus standard first-line pharmacological management for newly diagnosed immune thrombocytopaenia (ITP) in children: study protocol
}

To cite: Shimano KA, Grace RF, Despotovic JM, et al. Phase 3 randomised trial of eltrombopag versus standard first-line pharmacological management for newly diagnosed immune thrombocytopaenia (ITP) in children: study protocol. BMJ Open 2021;11:e044885. doi:10.1136/ bmjopen-2020-044885

- Prepublication history and additional supplemental material for this paper are available online. To view these files, please visit the journal online. (http://dx.doi.org/10.1136/ bmjopen-2020-044885).

Received 16 September 2020 Accepted 06 August 2021

Check for updates

(C) Author(s) (or their employer(s)) 2021. Re-use permitted under CC BY-NC. No commercial re-use. See rights and permissions. Published by BMJ.

For numbered affiliations see end of article.

Correspondence to Dr Kristin A Shimano; kristin.shimano@ucsf.edu

\section{ABSTRACT}

Introduction Immune thrombocytopaenia (ITP) is an acquired disorder of low platelets and risk of bleeding. Although many children can be observed until spontaneous remission, others require treatment due to bleeding or impact on health-related quality of life. Standard firstline therapies for those who need intervention include corticosteroids, intravenous immunoglobulin and anti-D globulin, though response to these agents may be only transient. Eltrombopag is an oral thrombopoietin receptor agonist approved for children with chronic ITP who have had an insufficient response to corticosteroids, intravenous immunoglobulin or splenectomy. This protocol paper describes an ongoing open-label, randomised trial comparing eltrombopag to standard first-line management in children with newly diagnosed ITP.

Methods and analysis Randomised treatment assignment is $2: 1$ for eltrombopag versus standard first-line management and is stratified by age and by prior treatment. The primary endpoint of the study is platelet response, defined as $\geq 3$ of 4 weeks with platelets $>50 \times 10^{9} /$ L during weeks $6-12$ of therapy. Secondary outcomes include number of rescue therapies needed during the first 12 weeks, proportion of patients who do not need ongoing treatment at 12 weeks and 6 months, proportion of patients with a treatment response at 1 year, and number of second-line therapies used in weeks 13-52, as well as changes in regulatory T cells, iron studies, bleeding, health-related quality of life and fatigue. A planned sample size of up to 162 randomised paediatric patients will be enrolled over 2 years at 20 sites.

Ethics and dissemination The study has been approved by the centralised Baylor University Institutional Review Board. The results are expected to be published in 2023. Trial registration number NCT03939637.

\section{INTRODUCTION}

Immune thrombocytopaenia (ITP) is the most common autoimmune cytopaenia in children, causing an often severely reduced platelet count, variable bleeding symptoms
Strengths and limitations of this study

- This is a multicentre randomised controlled trial that will provide valuable information about the efficacy of eltrombopag for newly diagnosed children with immune thrombocytopaenia (ITP).

- The primary endpoint is a well-defined definition of platelet response similar to that used in other ITP trials, while the secondary endpoints include important assessments of patient-related outcomes, including bleeding scores, fatigue and health-related quality of life, all of which may be more significant to patients than a platelet count alone.

- Correlative biology studies will allow exploration of biological differences between patients with ITP who are responders and non-responders, as well as biological mechanisms related to outcomes of specific interventions and the natural history of the disease in children receiving treatment.

- A limitation of this study is that patients who are managed with a close observation approach are not included or compared with those who require treatment.

- An additional limitation of this study is the lack of a uniform definition of 'requiring treatment,' which is left to the discretion of individual investigators; however, this allows for a 'real-world' approach and ultimately the potential for broader applicability of trial results to clinical practice.

and reduction in health-related quality of life (HRQoL) related to activity restrictions, frequent medical visits and interventions, anxiety from risk of bleeding and fatigue. ${ }^{1-3}$ In an era when the fields of haematology and immunology are advancing rapidly with the development of drugs targeted to underlying disease mechanisms, the available treatments for newly diagnosed ITP remain non-specific, 
Table 1 Administration, efficacy and potential side effects of standard therapies

\begin{tabular}{|c|c|c|c|c|}
\hline & Dosing & $\begin{array}{l}\text { Method of } \\
\text { administration }\end{array}$ & $\begin{array}{l}\text { Efficacy }^{37} \\
\text { (platelet }^{\star} \text { ) }\end{array}$ & Short-term potential side effects \\
\hline Prednisone & $\begin{array}{l}4 \mathrm{mg} / \mathrm{kg} / \text { day } \times 4-7 \\
\text { days } t\end{array}$ & Oral & 70\%-80\% & $\begin{array}{l}\text { Mood changes, hyperglycaemic, } \\
\text { hypertension, weight gain }\end{array}$ \\
\hline $\begin{array}{l}\text { Intravenous } \\
\text { immunoglobulin }\end{array}$ & $0.8-1 \mathrm{~g} / \mathrm{kg}$ & Intravenous & 70\%-80\% & $\begin{array}{l}\text { Headache, influenza-like symptoms, } \\
\text { allergic reaction, neutropaenia, } \\
\text { haemolytic anaemia } \\
\text { Black box: thrombosis, renal failure }\end{array}$ \\
\hline
\end{tabular}

${ }^{*}$ Definition of platelet response varies depending on study.

†A number of steroid regimens are used.

$\mathrm{DIC}$, disseminated intravascular coagulopathy.;

with no novel or targeted therapies introduced in the past 30 years. $^{4}$

Many children with ITP can be closely observed without treatment until they experience spontaneous remission. ${ }^{5}$ Others require pharmacological treatment for moderate bleeding or HRQoL limitations. While many therapies exist for treatment of chronic ITP, the treatment of newly diagnosed ITP is generally limited to close observation and three first-line medications: corticosteroids, intravenous immunoglobulin and anti-D globulin. Each of these agents has either undesirable side effects, challenging logistics of administration or both (table 1).

Furthermore, they act only transiently to raise the platelet count, and in children with ongoing ITP, the platelet count will decrease days to weeks after the medication is given.

Eltrombopag is an oral, small-molecule, non-peptide thrombopoietin receptor agonist (TPO-RA). It initiates thrombopoietin receptor signalling by interacting with the transmembrane domain of the receptor, inducing proliferation and differentiation of cells in the megakaryocytic lineage. Eltrombopag is currently approved for children ages $\geq 1$ year with chronic ITP who have had an insufficient response to corticosteroids, intravenous immunoglobulin or splenectomy. Safety and efficacy were established in the PETIT (Eltrombopag in Paediatric Patients with Thrombocytopaenia from Chronic ITP $)^{6}$ and PETIT2 $2^{7}$ trials. Forty per cent of patients who received eltrombopag vs $3 \%$ of patients randomised to placebo in the PETIT2 trial achieved the primary outcome, $\geq 6$ of 8 non-consecutive weeks with platelets $>50 \times 10^{9} / \mathrm{L}$ during weeks $5-12$ of therapy (OR $18.0,95 \%$ CI 2.3 to 140.9 ; $\mathrm{p}=0.0004){ }^{7}$ The drug was approved by the US Food and Drug Administration (FDA) in $2015^{8}$ and the European Medicines Agency in 2016 for children with chronic ITP. The majority of the literature to date evaluating the use of eltrombopag in the paediatric population has been in the setting of chronic ITP (table 2), although multicentre retrospective studies document that paediatric haematologists are using TPO-RAs off-label in some cases of newly diagnosed ITP. ${ }^{9}{ }^{10}$ Eltrombopag has been studied prospectively for adults with newly diagnosed ITP in two small single-centre trials. A single-arm study of dexamethasone in combination with 4 weeks of eltrombopag used upfront in adult patients with newly diagnosed ITP produced $100 \%$ response (platelets $>30 \times 10^{9} / \mathrm{L}$ ) at completion of therapy, and $66.7 \%$ relapse-free survival at 1 year, better outcomes than expected for comparable patients treated with steroids alone. ${ }^{11}$ In a second study, $76 \%$ of steroid-nonresponsive patients had a durable response to eltrombopag after 3 months of therapy. ${ }^{12}$ TPO-RAs may, therefore, be a safe and efficacious firstline therapy for newly diagnosed patients with ITP who require treatment.

The issue of long-term expense of a drug that costs thousands of dollars monthly and requires ongoing use has been both a practical and conceptual hurdle for use of TPO-RAs in children since their launch, but that thinking has evolved to consider that early in the course of illness the majority of cases of paediatric ITP will eventually resolve. While standard therapies like steroids, or when appropriate, observation only, are much less expensive than TPO-RAs, a few courses of intravenous immunoglobulin may rival the cost of a short course of eltrombopag, as well as require intravenous access and inpatient stays with associated complications that yield additional expense.

Eltrombopag has the potential to change the landscape of newly diagnosed ITP for children. The earliest randomised trial in children with newly diagnosed ITP took place in 1984 comparing prednisone to observation. ${ }^{13}$ Since that time only a handful of additional randomised trials have been conducted in this population, with the majority comparing intravenous immunoglobulin to anti-D immunoglobulin. ${ }^{14-21}$ The most recent randomised trial, conducted in 2018, was unable to show any long-term benefit to intravenous immunoglobulin compared with observation. ${ }^{22}$ No trial to date has investigated a novel agent for this patient population in a randomised manner. As an oral outpatient therapy 
Table 2 Published studies of eltrombopag in paediatric patients with ITP

\begin{tabular}{|c|c|c|c|}
\hline Paper* & Type of study & Patient population & Results \\
\hline Giordano et $a^{38}$ & $\begin{array}{l}\text { Retrospective multicentre } \\
\text { study }\end{array}$ & $\begin{array}{l}386 \text { children with chronic ITP enrolled } \\
\text { retrospectively at } 17 \text { sites }\end{array}$ & $\begin{array}{l}\text { Prevalence of eltrombopag use was } 19 \% \text { in } \\
\text { patients with chronic ITP }\end{array}$ \\
\hline Koca Yozgat et al ${ }^{9}$ & $\begin{array}{l}\text { Retrospective multicentre } \\
\text { study }\end{array}$ & $\begin{array}{l}105 \text { children with chronic or acute refractory ITP } \\
\text { treated with epag }\end{array}$ & $\begin{array}{l}\text { Overall response rate was } 74 \% .27 .6 \% \\
\text { developed iron deficiency or iron deficiency } \\
\text { anaemia. }\end{array}$ \\
\hline Cheng et $\mathrm{a} \mathrm{l}^{39}$ & $\begin{array}{l}\text { Single-centre } \\
\text { observational study }\end{array}$ & $\begin{array}{l}20 \text { patients with severe chronic ITP treated with } \\
\text { epag }\end{array}$ & The durable response rate was $70 \%(14 / 20)$ \\
\hline Suntsova et $a l^{41}$ & $\begin{array}{l}\text { Single-centre } \\
\text { retrospective analysis }\end{array}$ & $\begin{array}{l}23 \text { patients with chronic ITP who failed first } \\
\text { TPO-RA. } 10 \text { patients switched to epag. }\end{array}$ & $\begin{array}{l}\text { Response rates after switching TPO-RAs } \\
\text { were } 80 \% \text { (romi } \rightarrow \text { epag) and } 62 \% \text { (epag } \\
\rightarrow \text { romi) }\end{array}$ \\
\hline $\begin{array}{l}\text { Tumaini Massaro } \\
\text { et } a l^{42}\end{array}$ & Meta-analysis & $\begin{array}{l}\text { Five randomised controlled trials with total of } \\
261 \text { paediatric patients. } 159 \text { treated with epag. }\end{array}$ & TPO-RAs superior to placebo \\
\hline Grace et $a l^{44}$ & $\begin{array}{l}\text { Multicentre prospective } \\
\text { observational study }\end{array}$ & $\begin{array}{l}120 \text { children with ITP starting second-line } \\
\text { therapies. } 20 \text { patients treated with eltrombopag. }\end{array}$ & $\begin{array}{l}\text { Oral agents, including eltrombopag, were } \\
\text { chosen for ease of administration and } \\
\text { expected adherence }(p<0.001)\end{array}$ \\
\hline Leblebisatan et al ${ }^{45}$ & Single-arm study & 19 patients with chronic ITP & $\begin{array}{l}58 \% \text { of patients responded with either } \\
\text { increased platelet counts or decreased } \\
\text { bleeding }\end{array}$ \\
\hline Zhang et $a^{46}$ & $\begin{array}{l}\text { Indirect-comparison } \\
\text { meta-analysis }\end{array}$ & $\begin{array}{l}\text { Five randomised controlled trials with total of } \\
261 \text { paediatric patients. } 159 \text { treated with epag. }\end{array}$ & $\begin{array}{l}\text { Epag and romi similar in efficacy and } \\
\text { safety, but decreased bleeding w/ epag. }\end{array}$ \\
\hline Guo et $\mathrm{al}^{47}$ & Meta-analysis & $\begin{array}{l}\text { Seven randomised controlled trials with total of } \\
345 \text { paediatric patients. } 159 \text { treated with epag. }\end{array}$ & TPO-RAs superior to placebo \\
\hline Zhang et $a l^{48}$ & Systematic review & $\begin{array}{l}\text { Five randomised controlled trials with total of } \\
261 \text { paediatric patients. } 159 \text { treated with epag. }\end{array}$ & $\begin{array}{l}\text { Overall response and durable platelet } \\
\text { response increased in TPO-RAs versus } \\
\text { placebo }\end{array}$ \\
\hline Bussel et $a l^{6}$ & Multicentre RCT & $\begin{array}{l}82 \text { patients with ITP }>6 \text { months who had } \\
\text { received at least one prior treatment }\end{array}$ & $\begin{array}{l}62 \% \text { (vs } 32 \% \text { placebo) achieved platelet } \\
\text { count }>50 \times 1\end{array}$ \\
\hline Ramaswamy et al ${ }^{49}$ & $\begin{array}{l}\text { Multicentre retrospective } \\
\text { study }\end{array}$ & $\begin{array}{l}33 \text { paediatric patients with ITP who had received } \\
\text { at least one prior treatment; } 12 \text { received } \\
\text { eltrombopag }\end{array}$ & $\begin{array}{l}75 \% \text { achieved platelet counts } \geq 50 \mathrm{k} \text { and } \\
\geq 20 \mathrm{k} \text { above baseline for two consecutive } \\
\text { weeks }\end{array}$ \\
\hline
\end{tabular}

'Search was performed in PubMed using terms 'eltrombopag' and either 'paediatric' or 'children'. Clinical trials and meta-analyses were included. Papers were excluded if the patient population was anything other than paediatric patients with ITP, or if they included fewer than 10 patients treated with eltrombopag.

HRQoL, health-related quality of life; ITP, immune thrombocytopaenia; KIT, Kids ITP Tool; RCT, randomised controlled trial; TPO-RA, thrombopoietin receptor agonist.

which can be continued until ITP has remitted, eltrombopag has clear benefits over the transiently effective current first-line options. In addition, eltrombopag may have fewer side effects than standard therapies. However, the early response rate in the newly diagnosed setting is not known. We, therefore, describe here our design of an ongoing randomised trial investigating the up-front use of eltrombopag in paediatric patients with ITP: PINES (Paediatric ITP Newly diagnosed patients Eltrombopag vs Standard therapy) Study. The trial was FDA-approved in January 2019, and the first site opened to enrollment in May 2019. With this publication, we aim to provide researchers and funding agencies with early-stage information about this novel clinical trial which contributes to the gap in randomised trials for patients with paediatric newly diagnosed ITP. Ultimately, we hope that this will allow for transparency and collaboration with other research consortiums, as well as dissemination 
of knowledge about the study to patients not treated at PINES sites, which may encourage them to explore engagement in research protocols with their physicians.

\section{METHODS AND ANALYSIS}

\section{Study objectives and hypothesis}

The primary objective of the trial is to determine if the proportion of patients with a platelet response is significantly greater in patients with newly diagnosed ITP treated with eltrombopag than those treated with standard first-line pharmacological treatment. The primary endpoint, platelet response, is defined as $\geq 3$ of 4 non-consecutive weeks with platelets $>50 \times 10^{9} / \mathrm{L}$ during weeks 6-12 of therapy. We hypothesise that children with newly diagnosed ITP treated with eltrombopag will have an increased likelihood of a sustained platelet response as compared with those treated with standard therapy. The endpoints and statistical analysis plans of the primary and secondary objectives are listed in table 3 .

Table 3 Protocol endpoints and statistical analysis plans

\section{Outcome (endpoint) statistical plan}

\section{Efficacy}

1. Platelet response, defined as $\geq 3$ of 4 non-consecutive weeks with platelets $>50 \times 10^{9} / \mathrm{L}$ during weeks $6-12$ of therapy

Group sequential analyses, with three 'looks' at the data: two interim analyses (for efficacy and futility), and a final analysis (for efficacy), using a two-sided z-test with alpha $=0.05$ (ie, a one-sided $z$-test with alpha $=0.025)$, to compare the two arms in terms of the proportion of patients who have a platelet response

2. Cumulative number of rescue therapies needed during Student's t-test will be used to compare the two treatment arms the first 12 weeks of treatment
3. Platelet response during weeks $6-12$ of therapy in
Observed proportion and $95 \% \mathrm{Cl}$ will be calculated patients who required a rescue treatment during weeks $1-2$ of study

4. No further need for treatment after 12 weeks and $6 \quad \chi^{2}$ test will be used to compare the two treatment arms months of study

5. Treatment response* at 1 year of study

$\chi^{2}$ test will be used to compare the two treatment arms

6. No of second-line therapies used in weeks 13-52 Student's t-test will be used to compare the two treatment arms

\section{Safety}

7. Abnormal liver function tests† (LFTs) in patients with Frequency and proportion (with $95 \% \mathrm{Cl}$ ) of patients with abnormal LFTs will be calculated newly diagnosed ITP treated with eltrombopag.

8. Incidence of adverse events and serious adverse events
Adverse events will be coded by MedDRA classification term. Adverse events and serious adverse events will be tabulated by treatment group, including the number of patients for whom the event occurred, the rate of occurrence and the severity and relationship to study drug. If a patient experiences the same toxicity multiple times, a patient will be counted only once for a given toxicity at the maximum grade.

9. Iron indicesł at 12 weeks, 6 months and 1 year $\quad \begin{aligned} & \text { Two-sided Student's t-test will be used to compare iron indices at } 12 \text { weeks, } 6 \text { months and } 1 \\ & \text { year between the two arms }\end{aligned}$

\section{Patient-related outcomes assessment}

\section{Proportion of patients with poor bleeding scores $\quad \chi^{2}$ test will be used to compare the two treatment arms} (WHO Bleeding Scale $\geq 2$ or Modified Buchanan Score $\geq 3$ ) at 1, 2, 3, 4, 12 weeks and 1 year

11. Change in health-related quality of life from (A) baseline to 1 week, (B) baseline to 4 weeks, (C) baseline to 12 weeks, and, (D) baseline to 1 year, as measured by the parent-proxy report of the Kids ITP tools (KIT)

12. CF atigue at 1 week, 4 weeks, 12 weeks, and 1 year as measured by the parent-proxy report of the Hockenberry Fatigue Scale-Parent

KIT scores will be calculated per the methods described in Klaassen et al. ${ }^{29}$ Spaghetti plots will be used to visualise the KIT scores over time per patient by treatment arm.

Two-sided Student's t-test will be used to compare the percentage change from baseline in KIT overall score at 1 week, 4 weeks, 12 weeks and 1 year between the two arms.

Fatigue scores will be calculated per the methods described in Hockenberry et al. ${ }^{30}$ Spaghetti plots will be used to visualise the scores over time per patient by treatment arm. A two-sided Student's t-test will be used to compare the percentage change from baseline at 1 week, 4 weeks, 12 weeks and 1 year between the two arms.

\section{Biology}

13. Change in percentage of $\mathrm{CD} 4^{+} 25^{+} \mathrm{Foxp}^{+}$regulatory Student's t-test will be used to compare the two treatment arms $T$ cells as a proportion of CD4 cells (A) from baseline to 12 weeks; and (B) from baseline to 1 year

${ }^{*}$ Complete response (CR) is defined as a platelet count $\geq 150 \times 10^{9} / \mathrm{L}$, primary remission at 1 year is defined as $\mathrm{CR}$ at 1 year with no second-line agents required and $\geq 3$ months after discontinuing most recent platelet active medication, disease resolution at 1 year is defined as $\mathrm{CR}$ at 1 year $\geq 3$ months after discontinuing most recent platelet active medication (patient may have received a second-line therapy, excluding rituximab or splenectomy), disease stability at 1 year is defined as platelets $\geq 50 \times 10^{9} / \mathrm{L}$ but $<150 \times 10^{9} / \mathrm{L} \geq 3$ months after discontinuing most recent platelet active medication.

†Alanine transaminase (ALT) $\geq 3 x$ upper limit of normal (ULN) in patients with normal baseline, ALT $\geq 3 x$ baseline or $\geq 5 x$ ULN (whichever is lower) in patients with abnormal baseline, ALT $\geq 3 \times$ ULN and bilirubin $\geq 1.5 \times$ ULN ( $>35 \%$ direct).

ҒIron, total iron binding capacity, transferrin saturation, ferritin, mean corpuscular volume and haemoglobin.

ITP, immune thrombocytopaenia; MedDRA, Medical Dictionary for Regulatory Activities.; 


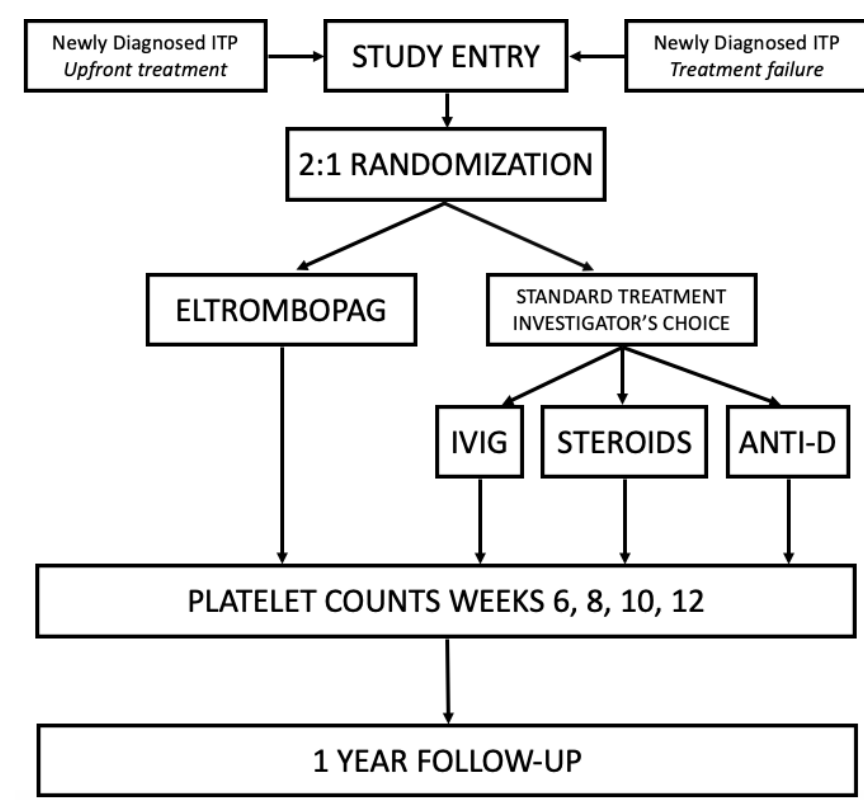

Figure 1 Schematic diagram of study entry, randomisation, primary endpoint evaluation and follow-up. ITP, immune thrombocytopaenia; IVIG, intravenous immunoglobulin.

In addition, exploratory objectives will include comparisons by treatment arm of other platelet-related endpoints, patient-related outcomes and cost of therapy. By obtaining data on patient-related outcomes such as HRQoL, we will be able to assess the potential impact of differences in drug delivery such as dietary restrictions, need for daily medication administration and potential impact of infusion therapy.

\section{Overview of study design and oversight}

The PINES Study is a national, multicentre, randomised, open-label, standard therapy-controlled trial. The study was designed to align with usual care for children with newly diagnosed ITP (figure 1). The screening period occurs from the time of diagnosis up until 3 months from the first low platelet count. Randomisation and initiation of treatment occurs at the baseline visit, and follow-up visits occur at week 1 , and 1, 3, 6, and 12 months from enrollment. Biweekly platelet counts are obtained from baseline through week 12. Patients will be followed for a total of 1 year from enrollment. Planned study visits and assessments are outlined in table 4 .

The study is being conducted at 20 national sites through the Pediatric ITP Consortium of North America (ICON). Participating sites are listed on ClinicalTrials. gov.

The trial is designed and led by a steering committee that includes academic investigators from ICON and statisticians from Dana-Farber/Boston Children's Cancer and Blood Disorders Centre, the coordinating centre for ICON. The steering committee will ensure transparent management of the study, recommend and approve study modifications, and develop recommendations for publications of study results. The trial is operated under an Investigational New Drug (IND) held by Baylor College of Medicine, cross-filed with Novartis. Novartis is providing funding for this investigator-initiated trial and supplies the drug used on the eltrombopag arm. An independent data safety monitoring committee (DSMC) monitors patient safety and outcomes at intervals during the study and makes recommendations to the steering committee regarding ongoing trial conduct. The protocol was reviewed and approved by regulatory authorities, a central institutional review board at Baylor College of Medicine, and institutional review boards at individual institutions. This clinical trial protocol follows the Standard Protocol Items: Recommendations for Interventional Trials (SPIRIT) guidelines ${ }^{23}$ (see SPIRIT checklist in online supplemental document 1).

\section{Study population, randomisation and stratification}

The study population includes children ages 1 to $<18$ years with newly diagnosed ITP $(<3$ months from first abnormal platelet count). At time of study screening, patients must have a platelet count $<30 \times 10^{9} / \mathrm{L}$ and

Table 4 Outline of study assessments

\begin{tabular}{|c|c|c|c|c|c|c|c|c|c|c|c|c|}
\hline & Day 0 & 72 hours & w1 & w2 & w3 & w4 & w6 & w8 & w10 & w12 & 6 months & 1 year \\
\hline Physical exam & $x$ & & $x$ & & & $x$ & & & & $x$ & $x$ & $x$ \\
\hline Bleeding assessment & $x$ & & $x$ & $x$ & $x$ & $x$ & & & & $x$ & & $x$ \\
\hline Hockenberry Fatigue Score & $x$ & & $x$ & & & $x$ & & & & $x$ & & $x$ \\
\hline PROMIS & $x$ & & & & & & & & & $x$ & & $x$ \\
\hline Platelet count & $x$ & $x$ & $x$ & $x$ & & $x$ & $x$ & $x$ & $x$ & $x$ & $x$ & $x$ \\
\hline Iron studies & $x$ & & & & & & & & & $x$ & & $x$ \\
\hline Tregs & $x$ & & & & & & & & & $x$ & & $x$ \\
\hline Samples for banking (optional) & $x$ & & & & & & & & & $x$ & & $x$ \\
\hline
\end{tabular}

.HRQOL, health-related quality of life; KIT, Kids ITP Tool; PROMIS, patient-reported outcomes measurement information system; Tregs, regulatory T cells; w, week. 
require pharmacological treatment from the perspective of the treating clinician. A platelet count of $<30 \times 10^{9} / \mathrm{L}$ was selected in order to provide a real-world approach to treatment in which patients often receive treatment based not on a platelet count threshold but rather for bleeding history or secondary to disease impact on HRQoL, both of which may occur at a higher platelet count. Recognising that there will be variation in physician practice with regards to treatment as well as heterogeneity in the reasons for treatment (bleeding, platelet count, HRQoL, etc.) we have elected to apply randomised trial design to help balance this variability between the two treatment groups. Patients who have previously received a TPO-RA are excluded. As we do not want to impede appropriate critical care management, patients with severe bleeding, defined as overall grade 4 or 5 bleeding, ${ }^{24}$ or bleeding requiring emergent treatment will be excluded. Patients are excluded if they have known secondary ITP (eg, due to lupus, common variable immunodeficiency or autoimmune lymphoproliferative disorder). Additional exclusion criteria are outlined in online supplemental table 1. All patients and/or their parents or legal guardians must sign a written informed consent and assent when applicable.

Patients who meet all of the inclusion and none of the exclusion criteria will be enrolled and randomly assigned to receive eltrombopag or standard therapy in a 2:1 ratio. Central randomisation at study enrollment will occur via the online InForm system, with randomisation allocation delivered only to the enrolling site study staff. Randomisation will use blocking and will be stratified by the age of the patient ( 1 to $<6,6$ to $<12$ and 12 to $<18$ years) and by prior treatment status. 'Upfront treatment' refers to patients within 10 days of ITP diagnosis who have not received previous pharmacological treatment. This allows for a reasonable window from time of diagnosis to enrollment as well as for proper time to confirm the diagnosis and rule out other transient causes of thrombocytopaenia. The 'treatment failure' stratum is for patients who have received standard initial management (observation $>10$ days, intravenous immunoglobulin, anti-D immunoglobulin or corticosteroids) and continue to have platelets $<30 \times 10^{9} / \mathrm{L}$. A patient who initially responded to treatment but whose response wanes and platelets fall below $30 \times 10^{9} / \mathrm{L}$ will be considered to have a 'treatment failure'.

\section{Eltrombopag regimen}

Patients randomised to eltrombopag will start at a dose based on age per the manufacturer label dosing for drug initiation. Children ages $1-5$ years will begin $25 \mathrm{mg}$ once daily, and children $\geq 6$ years will begin at a dose of $50 \mathrm{mg}$ once daily ( $25 \mathrm{mg}$ once daily for patients of East-Asian ethnicity). Patients will receive education regarding proper administration and dietary restrictions, and medication compliance will be queried at each study visit. Eltrombopag dose adjustments are made in response to platelet counts, with a goal of maintaining a platelet count $50-200 \times 10^{9} / \mathrm{L}$, with a maximum dose of $75 \mathrm{mg}$ once daily (online supplemental table 2). A modification from the dosing label was made for maintenance dose adjustment based on investigators' clinical experience with rebound thrombocytopaenia when eltrombopag is held for high platelet counts. Daily eltrombopag will be continued for 12 weeks, unless platelets do not rise $\geq 30 \times 10^{9} / \mathrm{L}$ after 4 weeks at the maximum daily dose of $75 \mathrm{mg}$.

Patients randomised to the eltrombopag arm who respond will be eligible to continue the treatment throughout the 1-year duration of study participation, with guidelines given for dose adjustments during weeks 13-52 (online supplemental table 2). For patients who have completed 12 weeks of eltrombopag and maintain a platelet count $\geq 100 \times 10^{9} / \mathrm{L}$, eltrombopag will be weaned, by dose reducing $12.5 \mathrm{mg}$ every 2 weeks (with investigators exercising clinical judgement with respect to wean in the context of the patient's status, including intercurrent infections). Patients who have been unable to wean off of eltrombopag by 1 year will discontinue study drug, and continuation on commercially available eltrombopag at that point is at the discretion of the investigator.

\section{Standard therapy regimen}

Subjects randomised to the standard therapy arm will receive one of three treatments at the discretion of the treating physician. Patients previously treated with standard management prior to study entry must be treated with a different agent than their original failed agent. For example, a patient who did not respond to steroids could receive either intravenous immunoglobulin or anti-D if randomised to the standard treatment arm. Investigators may choose among the following treatment options: (1) intravenous immunoglobulin $1 \mathrm{~g} / \mathrm{kg} \times 1$ dose, ${ }^{25}$ (2) prednisone/prednisolone $4 \mathrm{mg} / \mathrm{kg}$ /day (max $120 \mathrm{mg}$ / day) $\times 4$ days $^{26}$ or (3) anti-D immunoglobulin $75 \mu \mathrm{g} / \mathrm{kg}$ $\times 1$ dose. $^{25}$ No steroids for premedication or adjunctive therapy may be administered with intravenous immunoglobulin or anti-D immunoglobulin.

\section{Efficacy outcomes}

The primary endpoint is binary, with each patient classified as either a platelet responder or a platelet nonresponder. Platelet response is defined as $\geq 3$ of 4 weeks with platelets $>50 \times 10^{9} / \mathrm{L}$ (whereby the 3 weeks are not required to be consecutive) during weeks 6-12 of therapy. The primary endpoint for this study was initially defined as $\geq 6$ of 8 weeks platelets $>50 \times 10^{9} / \mathrm{L}$, and was chosen in part because it is a previously defined primary endpoint in a prior ITP study. ${ }^{7}$ The COVID-19 pandemic introduced safety and logistics concerns for the conduct of clinical research, however, particularly with respect to study assessments done for research purposes only that would require additional exposure to clinic or lab settings during shelter-in-place restrictions. The study DSMC, therefore, recommended a change to the primary endpoint to a clinically equivalent definition that would require fewer lab assessments as a measure to prioritise patient safety during the pandemic and in 
order to minimise missing data if subjects were unable to complete a study assessment due to pandemic-related safety concerns. The primary endpoint represents a clinically relevant outcome in the newly diagnosed setting, as patients who are being treated because of bleeding symptoms or risk may benefit from a more sustained response during this time period, rather than repeated drops in platelet counts after transient responses to therapy. While rescue therapies (steroids, intravenous immunoglobulin or anti-D globulin) are permitted during the study, patients who require a rescue medication at any time within the first 12 weeks of therapy will be categorised as a non-responder.

\section{Secondary outcomes}

Additional response outcomes include the number of rescue therapies needed during the first 12 weeks, platelet response during weeks 6-12 of study in patients who required a rescue treatment during that time, proportion of patients who do not need ongoing treatment at 12 weeks and 6 months, proportion of patients with a treatment response at 1 year after study enrollment and the number of second-line therapies (treatments other than prednisone, intravenous immunoglobulin and anti-D globulin thought to be active in the treatment of ITP) used in weeks 13-52. Safety analyses will examine the proportion of patients with abnormal liver function tests in patients with newly diagnosed ITP treated with eltrombopag, and the proportion of patients with adverse events and serious adverse events (SAEs) by treatment arm. Furthermore, we will investigate changes in iron indices (serum iron, total iron binding capacity (TIBC), transferrin saturation, ferritin, mean corpuscular volume (MCV) and haemoglobin) given the chelation properties of eltrombopag. ${ }^{97}$ Secondary analyses will also include comparison of patient-related outcomes for patients treated with eltrombopag versus those treated with standard first-line agents. This includes comparison of significant bleeding (WHO Bleeding Scale $\geq 2^{28}$ or Modified Buchanan Score $\geq 3^{24}$ ), change in HRQoL measured by the Kids ITP Tool, ${ }^{29}$ and fatigue as measured by the parentproxy report of the Hockenberry Fatigue Scale-Parent. ${ }^{30}$

\section{Correlative biology studies}

Age and duration of symptoms at diagnosis are known to be associated with resolution of ITP, ${ }^{31}$ but other biological factors that predispose some patients to resolution of their ITP and others to a more chronic course are not known. It is also unknown whether the development of chronic ITP could be prevented by intervention with a TPO-RA early in a patient's course. A subset of patients with chronic ITP maintained increased platelets after discontinuation of treatment with TPO-RAs. ${ }^{32-34}$ Because of the implication of Tregs in the pathogenesis of ITP and the potential immunomodulatory effects of TPO-RAs, early use of eltrombopag may have a positive impact on the number of patients who develop chronic disease. For this reason, we will evaluate the change in percentage of $\mathrm{CD}^{+} 25^{+} \mathrm{Foxp}^{+}$Tregs in patients treated with eltrombopag compared with those treated with standard first-line agents. Additionally, there are likely biological factors which influence response to TPO-RAs and other therapies that are not yet understood. Identification of biomarkers of treatment response could lead to a personalised approach to therapy, targeted to an individual patient's disease biology. If consent is obtained for optional studies, baseline DNA samples and baseline and serial RNA samples will be banked for future correlative biology studies.

\section{Exploratory outcomes}

Additional analyses will include comparison of patients treated with eltrombopag versus those treated with standard first-line agents using International Working Group platelet-specific endpoints. ${ }^{35}$ Lastly, we plan to conduct a cost analysis of therapy between the two treatment arms, recognising the large cost difference between some current first-line therapy agents such as corticosteroids and eltrombopag.

\section{Sample size and statistical plan}

A total of up to 162 patients will be enrolled. For the primary objective, all randomised patients will be analysed in an intent-to-treat (ITT) analysis of response rate for the primary objective. A patient is considered 'non-informative' if he withdraws from protocol therapy and data submission prior to the 6-week platelet assessment. Non-informative patients will be classified as nonresponders, and both informative and non-informative patients will be included in the ITT analysis. Noninformative patients could dilute our ability to detect a treatment effect; therefore, additional patients will be randomised to make up for the diluting effect. A conservatively high estimate of $9 \%$ of patients are anticipated to be non-informative. To obtain at least 147 informative randomised patients, we plan to enrol and randomise up to 15 additional patients ( 10 and 5 for the eltrombopag and standard treatment arms, respectively) for a total of up to 162. At an anticipated enrollment rate of 90 patients per year, the total accrual duration is expected to be 2 years, plus 1-year follow-up on the last patient, for a total study duration of 3 years.

The primary objective will be addressed by monitoring for evidence of efficacy or lack of efficacy (futility) using group sequential analyses, with three 'looks' at the data (after one-third, two-thirds and full accrual). In each analysis, a two-sided z-test will be used to compare the two arms in terms of the proportion of patients who have a platelet response. We will reject the null hypothesis if the upper (efficacy) monitoring boundary is crossed; in this case, it will be reasonable to conclude that the platelet response is significantly greater in patients treated with eltrombopag than standard first-line treatments. If the lower (futility) monitoring boundary is crossed in either of the two interim analyses, we will have significant evidence that eltrombopag is not more efficacious than standard 
first-line treatments, and the trial will be stopped early for futility. The overall type 1 error is preserved at 0.05 , or 0.025 in a one-sided test. The sample size of 147 informative patients will provide $81.4 \%$ power to detect an absolute difference of $25 \%$ in the proportion of patients who are platelet responders, assuming a response rate of $75 \%$ with eltrombopag and $50 \%$ with standard first-line treatments, using a two-sided z-test with alpha $=0.05$ (ie, a one-sided $z$-test with alpha $=0.025$ ).

\section{DISCUSSION}

ICON is a group of 50 paediatric haematology centres in the USA, Canada and Mexico participating in collaborative research efforts dedicated to improving the understanding, treatment and quality of life of paediatric patients with ITP. The consortium was established in 2012 and has previously completed a prospective observational trial of patients starting second-line therapies for ITP. PINES is the Consortium's first prospective investigational trial for newly diagnosed ITP, initiated in response to a need for alternative up-front treatment options. While many children with ITP can be safely observed while waiting for spontaneous resolution of their disease, for those who require intervention, treatment options are limited and may only transiently increase the platelet count without achieving a sustained response. An optimal therapy for patients who do warrant treatment for repeated bleeding episodes or poor quality of life would be an easy-toadminister medication with a tolerable side effect profile that produces a sustained response until resolution.

The primary endpoint of PINES, $\geq 3$ of 4 weeks with platelets $>50 \times 10^{9} / \mathrm{L}$ during weeks $6-12$ of therapy, is a clinically relevant measure of platelet response suggesting sustained response to therapy. It also parallels a previously established endpoint for eltrombopag in paediatric ITP used in the PETIT2 trial. ${ }^{7}$ With a goal of 162 randomised patients, the study is powered to detect an improvement of $25 \%$ in the proportion of patients who are platelet responders in the eltrombopag arm compared with standard first-line treatments. Secondary platelet endpoints include treatment response at 1 year, with response definitions based on platelet count and time since most recent platelet active medication. Additional plateletspecific endpoints are included in exploratory objectives with International Working Group defined endpoints with a goal of being able to compare across studies. ${ }^{35}$ Another strength of this study is the collection of patientrelated outcomes data. The 2019 American Society of Hematology guidelines stressed that for prioritised outcomes such as bleeding and HRQoL there is a paucity of necessary data to guide clinical practice. ${ }^{5}$ It is critical in any contemporary interventional ITP study to assess bleeding and HRQoL in addition to platelet response, as bleeding severity, platelet count and HRQoL scores are uncorrelated independent outcomes, each of which may impact treatment decisions. ${ }^{36}$
The experimental design of the trial is intended to allow for maximum clinical discretion on the part of the treating investigator, with a 'real-world' approach to decision making. Patients are eligible to enrol if they require pharmacological treatment (for whatever reason) in the opinion of the treating haematologist, as long as they do not have severe bleeding that requires emergent intervention or concomitant therapy to achieve a rapid rise in platelet count. If the patient is randomised to the standard therapy arm, the investigator may choose among three standard treatments at protocol-specified doses. Rescue medications are allowed throughout the study, and after week 12 of the study, therapy in the standard arm or for non-responders in the eltrombopag arm is at the discretion of the investigator.

Because it is anticipated that the majority of patients will have remission of their ITP before the end of the 1-year duration of study participation, protocol-prescribed adjustments of eltrombopag during weeks $13-52$ of the study lead to more aggressive weaning than would result from the manufacturer recommendations for dose adjustment in the setting of chronic ITP.

In general, paediatric patients with newly diagnosed ITP have very favourable outcomes, and as such we are loath to expose these patients to any undue risk. Because eltrombopag already has an established safety profile in the paediatric population and is an FDA-approved treatment for paediatric patients with chronic ITP, we are reassured that this is a safe therapy for patients with newly diagnosed ITP. However, because safety is paramount, we have chosen to be particularly conservative with exclusion criteria, and we have chosen stringent cut-offs of transaminases and bilirubin. Iron deficiency has been reported in two retrospective series of patients treated with eltrombopag, ${ }^{927}$ and we will be able to follow this larger cohort of patients prospectively to better evaluate the incidence of this potential side effect.

A prospective randomised trial presents a unique opportunity to explore biological differences in disease between treatment responders and non-responders as well as biological outcomes of specific interventions. Tregs play a role in the pathogenesis of ITP, but it is not clear what impact the interactions of medical therapies with Tregs have on response, and following these over time may add to our understanding of the underlying biology of ITP development and resolution. Finally, through banking DNA and RNA samples for future studies, we anticipate possible identification of genes associated with response to therapies or RNA expression changes that correlate with disease activity that may improve our understanding of how to optimally treat paediatric patients with ITP.

We describe an in-process randomised clinical trial comparing eltrombopag to standard therapy in the treatment of paediatric patients with newly diagnosed ITP. This is the first paediatric trial investigating the use of a TPO-RA for patients with newly diagnosed ITP and has the potential to transform our approach to treatment in this patient population. While the primary outcome of this 
study is sustained platelet response during weeks 6-12 of treatment, the clinical implications surpass platelet count alone. The possibility of a limited course of a TPO-RA in the newly diagnosed phase that could bridge the time to spontaneous resolution of disease may diminish bleeding episodes and improve quality of life for these patients.

\section{Patient and public involvement}

Patients were not directly involved in the design of this study, although the consortium meets regularly with ITP patient advocacy group members and leaders, including the Platelet Disorder Support Association (PDSA), in order to understand needs and priorities of the patients. The PDSA has disseminated information about the trial to its members via website, and results and lay summary will be provided to patient groups and the public after trial completion.

\section{ETHICS AND DISSEMINATION}

The study protocol, informed consent and assent forms, and surveys have been approved by the central IRB at Baylor University/Texas Children's Hospital (see online supplemental document 2). The study protocol was approved on 28 January 2019, and this manuscript details the protocol in the latest version V.4.1 approved on 26 April 2021.

Participating consortium sites have either executed a reliance agreement to rely on the central IRB or have obtained approval from their local IRBs. Data management for the study is through an InForm database managed by the data coordinating centre, Boston Children's Hospital. Data will be entered electronically at the participating sites. Study sites will be monitored at 6-month intervals by a team from the data coordinating centre, with audits to review and verify data recorded on case report forms (CRFs) against source documents. Deidentified study information and study documents are sent via secure file transfer systems.

SAEs are reported to the central IRB and local IRBs as well as to Novartis.

The trial design and rationale has been presented in poster form at a national meeting. Following trial completion, results of the study will be submitted for peer review for publication in a scientific journal. The writing committee will consist of members of the trial steering committee, site investigators and ICON consortium members. The full protocol and dataset will be publicly available on request after completion and publication of planned analyses.

\section{Author affiliations}

${ }^{1}$ UCSF Benioff Children's Hospital, San Francisco, California, USA

${ }^{2}$ Pediatrics, UCSF, San Francisco, California, USA

${ }^{3}$ Dana-Farber/Boston Children's Cancer and Blood Disorders Center, Boston,

Massachusetts, USA

${ }^{4}$ Texas Children's Hospital, Houston, Texas, USA

${ }^{5}$ Baylor College of Medicine, Houston, Texas, USA

${ }^{6}$ St. Jude Children's Research Hospital, Memphis, Tennessee, USA
${ }^{7}$ Children's Hospital of Eastern Ontario, Ottawa, Ontario, Canada

${ }^{8}$ Pediatrics, Emory University, Atlanta, Georgia, USA

${ }^{9}$ Aflac Cancer and Blood Disorders Center, Children's Healthcare of Atlanta, Atlanta, Georgia, USA

${ }^{10}$ Centre for Addiction and Mental Health, Toronto, Ontario, Canada

${ }^{11}$ Biostatistics, Harvard Medical School, Boston, Massachusetts, USA

${ }^{12}$ Pediatrics, Columbia University Medical School, New York, New York, USA

Acknowledgements The authors thank the members of the Platelet Disorder Support Association (PDSA) for their ongoing support of the ITP Consortium of North America, and PDSA President and CEO Caroline Kruse for her review of this manuscript. The authors also thank all of the ICON3/PINES investigators and site research teams.

Contributors The study was conceived of and designed by KAS, RFG, JD, EN, RJK, CB and CEN. CM and WL designed the statistical analysis. KAS, RFG and CEN wrote the original manuscript draft which was reviewed and revised by all the coauthors.

Funding PINES is a consortium investigator-initiated trial with funding and investigational drug provided by Novartis (award/grant number not applicable).

Competing interests KAS: Research funding: Novartis, Pfizer, Daiichi Sankyo, Alexion; Consultancies: Dova. RFG: Research funding: Novartis, Agios, Pfizer; Consultancies: Agios, Dova. JD: Research funding: Amgen, Novartis; Consultancies: Amgen, Novartis, Dova. EN: Advisory boards: Genentech, NovoNordisk, Novartis; Honoraria: Octapharma; DSMB service: Bayer, ApoPharma, Acceleron, Imara; Consultancies: Pfizer, Celgene. RJK: Speaker: Takeda, Biogen Canada LMT, Octapharma, Pfizer; Consultancies: Agios, Amgen, Hoffman-LaRoche, Takeda, NovoNordisk Canada. CM: Research funding: Novartis. WL: DSMB member: ArQule, Jubliant Draximage. CEN: Research funding: PDSA.

Patient consent for publication Not required.

Provenance and peer review Not commissioned; externally peer reviewed.

Supplemental material This content has been supplied by the author(s). It has not been vetted by BMJ Publishing Group Limited (BMJ) and may not have been peer-reviewed. Any opinions or recommendations discussed are solely those of the author(s) and are not endorsed by BMJ. BMJ disclaims all liability and responsibility arising from any reliance placed on the content. Where the content includes any translated material, BMJ does not warrant the accuracy and reliability of the translations (including but not limited to local regulations, clinical guidelines, terminology, drug names and drug dosages), and is not responsible for any error and/or omissions arising from translation and adaptation or otherwise.

Open access This is an open access article distributed in accordance with the Creative Commons Attribution Non Commercial (CC BY-NC 4.0) license, which permits others to distribute, remix, adapt, build upon this work non-commercially, and license their derivative works on different terms, provided the original work is properly cited, appropriate credit is given, any changes made indicated, and the use is non-commercial. See: http://creativecommons.org/licenses/by-nc/4.0/.

ORCID iD

Kristin A Shimano http://orcid.org/0000-0001-8371-1040

\section{REFERENCES}

1 Neunert C, Noroozi N, Norman G, et al. Severe bleeding events in adults and children with primary immune thrombocytopenia: a systematic review. J Thromb Haemost 2015;13:457-64.

2 Kumar M, Lambert MP, Breakey V, et al. Sports participation in children and adolescents with immune thrombocytopenia (ITP). Pediatr Blood Cancer 2015;62:2223-5.

3 Hill QA, Newland AC. Fatigue in immune thrombocytopenia. Br J Haematol 2015;170:141-9.

4 Stasi R, Newland AC. ITP: a historical perspective. Br J Haematol 2011;153:437-50.

5 Neunert C, Terrell DR, Arnold DM, et al. American Society of hematology 2019 guidelines for immune thrombocytopenia. Blood Adv 2019;3:3829-66.

6 Bussel JB, de Miguel PG, Despotovic JM, et al. Eltrombopag for the treatment of children with persistent and chronic immune thrombocytopenia (petit): a randomised, multicentre, placebocontrolled study. Lancet Haematol 2015;2:e315-25.

7 Grainger JD, Locatelli F, Chotsampancharoen T, et al. Eltrombopag for children with chronic immune thrombocytopenia (PETIT2): 
a randomised, multicentre, placebo-controlled trial. Lancet 2015;386:1649-58

8 Ehrlich LA, Kwitkowski VE, Reaman G, et al. U.S. food and drug administration approval summary: eltrombopag for the treatment of pediatric patients with chronic immune (idiopathic) thrombocytopenia. Pediatr Blood Cancer 2017;64:e26657.

9 Koca Yozgat A, Leblebisatan G, Akbayram S, et al. Outcomes of eltrombopag treatment and development of iron deficiency in children with immune thrombocytopenia in turkey. Turk $\mathrm{J}$ Haematol 2020;37:139-44.

10 Neunert C, Despotovic J, Haley K, et al. Thrombopoietin receptor agonist use in children: data from the pediatric ITP Consortium of North America ICON2 study. Pediatr Blood Cancer 2016;63:1407-13.

11 Gómez-Almaguer D, Herrera-Rojas MA, Jaime-Pérez JC, et al. Eltrombopag and high-dose dexamethasone as frontline treatment of newly diagnosed immune thrombocytopenia in adults. Blood 2014;123:3906-8.

12 Tripathi AK, Shukla A, Mishra S, et al. Eltrombopag therapy in newly diagnosed steroid non-responsive ITP patients. Int $\mathrm{J}$ Hematol 2014:99:413-7.

13 Buchanan GR, Holtkamp CA. Prednisone therapy for children with newly diagnosed idiopathic thrombocytopenic purpura. A randomized clinical trial. Am J Pediatr Hematol Oncol 1984:6:355-62.

14 Papagianni A, Economou M, Tragiannidis A, et al. Standarddose intravenous anti- $D$ immunoglobulin versus intravenous immunoglobulin in the treatment of newly diagnosed childhood primary immune thrombocytopenia. J Pediatr Hematol Oncol 2011;33:265-9.

15 Alioglu B, Ercan S, Tapci AE, et al. A comparison of intravenous immunoglobulin ( $2 \mathrm{~g} / \mathrm{kg}$ totally) and single doses of anti-D immunoglobulin at $50 \mu \mathrm{g} / \mathrm{kg}, 75 \mu \mathrm{g} / \mathrm{kg}$ in newly diagnosed children with idiopathic thrombocytopenic purpura: Ankara Hospital experience. Blood Coagul Fibrinolysis 2013;24:505-9.

16 Celik M, Bulbul A, Aydogan G, et al. Comparison of anti-D immunoglobulin, methylprednisolone, or intravenous immunoglobulin therapy in newly diagnosed pediatric immune thrombocytopenic purpura. J Thromb Thrombolysis 2013;35:228-33.

17 Son DW, Jeon I-sang, Yang SW, et al. A single dose of anti-D immunoglobulin raises platelet count as efficiently as intravenous immunoglobulin in newly diagnosed immune thrombocytopenic purpura in Korean children. $J$ Pediatr Hematol Oncol 2008;30:598-601.

18 Tarantino MD, Young G, Bertolone SJ, et al. Single dose of anti-D immune globulin at 75 microg/kg is as effective as intravenous immune globulin at rapidly raising the platelet count in newly diagnosed immune thrombocytopenic purpura in children. $J$ Pediatr 2006;148:489-94.

19 Shahgholi E, Vosough P, Sotoudeh K, et al. Intravenous immune globulin versus intravenous anti-D immune globulin for the treatment of acute immune thrombocytopenic purpura. Indian J Pediatr 2008;75:1231-5

20 Fujisawa $\mathrm{K}$, lyori $\mathrm{H}$, Ohkawa $\mathrm{H}$, et al. A prospective, randomized trial of conventional, dose-accelerated corticosteroids and intravenous immunoglobulin in children with newly diagnosed idiopathic thrombocytopenic purpura. Int J Hematol 2000;72:376-83.

21 Rosthøj S, Nielsen S, Pedersen FK. Randomized trial comparing intravenous immunoglobulin with methylprednisolone pulse therapy in acute idiopathic thrombocytopenic purpura. Danish I.T.P. Study Group. Acta Paediatr 1996;85:910-5.

22 Heitink-Pollé KMJ, Uiterwaal CSPM, Porcelijn L, et al. Intravenous immunoglobulin vs observation in childhood immune thrombocytopenia: a randomized controlled trial. Blood 2018;132:883-91.

23 Chan A-W, Tetzlaff JM, Altman DG, et al. Spirit 2013 statement: defining standard protocol items for clinical trials. Ann Intern Med 2013;158:200-7.

24 Buchanan GR, Adix L. Grading of hemorrhage in children with idiopathic thrombocytopenic purpura. J Pediatr 2002;141:683-8.

25 Kane I, Ragucci D, Shatat IF, et al. Comparison of intravenous immune globulin and high dose anti-D immune globulin as initial therapy for childhood immune thrombocytopenic purpura. Br J Haematol 2010;149:79-83.

26 Carcao MD, Zipursky A, Butchart S, et al. Short-Course oral prednisone therapy in children presenting with acute immune thrombocytopenic purpura (ITP). Acta Paediatr Suppl 1998;424:71-4.

27 Lambert MP, Witmer CM, Kwiatkowski JL. Therapy induced iron deficiency in children treated with eltrombopag for immune thrombocytopenia. Am J Hematol 2017;92:E88-91.
28 Fogarty PF, Tarantino MD, Brainsky A, et al. Selective validation of the who bleeding scale in patients with chronic immune thrombocytopenia. Curr Med Res Opin 2012;28:79-87.

29 Klaassen RJ, Blanchette VS, Barnard D, et al. Validity, reliability, and responsiveness of a new measure of health-related quality of life in children with immune thrombocytopenic purpura: the kids' ITP tools. J Pediatr 2007:150:510-5.

30 Hockenberry MJ, Hinds PS, Barrera P, et al. Three instruments to assess fatigue in children with cancer. J Pain Symptom Manage 2003;25:319-28.

31 Revel-Vilk S, Yacobovich J, Frank S, et al. Age and duration of bleeding symptoms at diagnosis best predict resolution of childhood immune thrombocytopenia at 3,6 , and 12 months. J Pediatr 2013;163:1335-9.

32 Ghadaki B, Nazi I, Kelton JG, et al. Sustained remissions of immune thrombocytopenia associated with the use of thrombopoietin receptor agonists. Transfusion 2013;53:2807-12.

33 González-López TJ, Pascual C, Álvarez-Román MT, et al. Successful discontinuation of eltrombopag after complete remission in patients with primary immune thrombocytopenia. Am J Hematol 2015;90:E40-3.

34 Wang X, Liu X, Wang L, et al. Successful discontinuation of eltrombopag in one child with refractory primary immune thrombocytopenia and literature review. Blood Coagul Fibrinolysis 2019;30:71-4.

35 Rodeghiero F, Stasi R, Gernsheimer T, et al. Standardization of terminology, definitions and outcome criteria in immune thrombocytopenic purpura of adults and children: report from an international Working group. Blood 2009;113:2386-93.

36 Neunert CE, Buchanan GR, Blanchette V, et al. Relationships among bleeding severity, health-related quality of life, and platelet count in children with immune thrombocytopenic purpura. Pediatr Blood Cancer 2009;53:652-4.

37 Provan D, Stasi R, Newland AC, et al. International consensus report on the investigation and management of primary immune thrombocytopenia. Blood 2010;115:168-86.

38 Giordano P, Lassandro G, Barone A, et al. Use of eltrombopag in children with chronic immune thrombocytopenia (ITP): a real life retrospective multicenter experience of the Italian association of pediatric hematology and oncology (AIEOP). Front Med 2020;7:66.

39 Cheng X, Yan K, Ma J, et al. Efficacy and safety of eltrombopag in the treatment of severe chronic immune thrombocytopenia in children of China: a single-center observational study. Int $J$ Immunopathol Pharmacol 2019;33:2058738419872120.

40 Grace RF, Shimano KA, Bhat R, et al. Second-line treatments in children with immune thrombocytopenia: effect on platelet count and patient-centered outcomes. Am J Hematol 2019;94:741-50.

41 Suntsova EV, Maschan AA, Baydildina DD, et al. Thrombopoietin receptor agonist switch in children with persistent and chronic severe immune thrombocytopenia: a retrospective analysis in a large tertiary center. Pediatr Blood Cancer 2019;66:e27704.

42 Tumaini Massaro J, Chen Y, Ke Z. Efficacy and safety of thrombopoietin receptor agonists in children with chronic immune thrombocytopenic purpura: meta-analysis. Platelets 2019;30:828-35.

43 Grainger JD, Blanchette VS, Grotzinger KM, et al. Health-related quality of life in children with chronic immune thrombocytopenia treated with eltrombopag in the petit study. $\mathrm{Br} J$ Haematol 2019:185:102-6.

44 Grace RF, Despotovic JM, Bennett CM, et al. Physician decision making in selection of second-line treatments in immune thrombocytopenia in children. Am J Hematol 2018;93:882-8.

45 Leblebisatan G, Kilinc Y, Cil M, et al. Eltrombopag for immune thrombocytopenic children in a single region. Indian $\mathrm{J} \mathrm{Hematol} \mathrm{Blood}$ Transfus 2018;34:707-10.

46 Zhang J, Liang Y, Ai Y, et al. Eltrombopag versus romiplostim in treatment of children with persistent or chronic immune thrombocytopenia: a systematic review incorporating an indirectcomparison meta-analysis. Sci Rep 2018;8:576.

47 Guo J-C, Zheng Y, Chen H-T, et al. Efficacy and safety of thrombopoietin receptor agonists in children with chronic immune thrombocytopenia: a meta-analysis. Oncotarget 2018;9:7112-25.

48 Zhang J, Liang Y, Ai Y, et al. Thrombopoietin-receptor agonists for children with immune thrombocytopenia: a systematic review. Expert Opin Pharmacother 2017;18:1543-51.

49 Ramaswamy K, Hsieh L, Leven E, et al. Thrombopoietic agents for the treatment of persistent and chronic immune thrombocytopenia in children. J Pediatr 2014;165:600-5. 